\title{
Daunorubicin Induced Steven-Johnson Syndrome: A Case Report
}

\author{
Preeti Shakya ${ }^{1}$ and Amit Sharma Nepal ${ }^{2}$ \\ ${ }^{1}$ BP Koirala Memorial Cancer Hospital \\ ${ }^{2}$ Tribhuvan University Institute of Medicine
}

December 28, 2020

\begin{abstract}
The association of Daunorubicin and Steven-Johnson Syndrome (SJS) has not been well-established. In this report, we describe a fatal case of a 2 years old boy with acute leukemia who developed Steven-Johnson Syndrome owing to Daunorubicin. Clinicians should consider the possible association of Daunorubicin with SJS.
\end{abstract}

\section{INTRODUCTION:}

According to the extent of widespread epidermal detachment, SJS/TEN is classified as SJS, SJS/TEN overlap, and TEN with less than 10\%,10\%-30\%, and 30\% of body surface area, respectively. The majority of cases of SJS/TEN are the results of a hypersensitive reaction to a drug, and the drugs most commonly associated with the diseases are anticonvulsants, sulfonamide antibiotics, antiretrovirals, non-steroidal antiinflammatory drugs (NSAIDs), allopurinol, and corticosteroids [1-4]. The potential association of anticancer agents with SJS/TEN has not been systematically investigated and has been inconsistently reported. Daunorubicin is an anthracycline antibiotic that has antineoplastic activity and is used in the therapy of acute leukemia and AIDS related Kaposi sarcoma. [5] Cutaneous side- effects of Daunorubicin are very rare. In this report, we describe a fatal case of a 2 years old boy with Precursor B Cell Acute Lymphoblastic Leukaemia who developed SJS owing to Daunorubicin.

Key words: Steven-Johnson Syndrome/ Toxic Epidermal Necrolysis, chemotherapy, Daunorubicin, hypersensiticvity reaction

\section{KEY CLINICAL MESSAGE:}

Clinicians should consider the possible association of Daunorubicin with SJS, administer it with caution and promptly evaluate all subsequently developing cutaneous reactions with high index of suspicion for Steven-Johnson Syndrome.

\section{CASE REPORT :}

A 2 years old male child diagnosed with Precursor B cell Acute Lymphoblastic Leukaemia/Lymphoma (ALL) underwent a multi-drug regimen remission induction chemotherapy as per the modified BerlinFrankfurt-Münster (BFM) 90 ALL protocol with Vincristine $1.4 \mathrm{mg} / \mathrm{m} 2 /$ dose intravenously once a week (D1,8), Prednisolone 40mg/m2/day orally daily (D1-8), L-Asparaginase 6000 Units $/ \mathrm{m} 2 /$ dose intravenously q.a.d from D2 (a total of 3 doses), Methotrexate $8 \mathrm{mg} /$ dose intrathecally once a week $(\mathrm{D} 1,8)$ and Daunorubicin $30 \mathrm{mg} / \mathrm{m} 2 /$ dose intraveneously on $\mathrm{D} 8$.

About two weeks after the initiation of first induction chemotherapy, the patient developed cutaneous erythema around the face extending to the chest with ulceration of mucosal surfaces of oropharynx which quickly progressed into confluent erythematous and necrotic eruption with blistering of the skin. 
On systemic examination, the patient was febrile and there was presence of vesicobullous lesions over the face and neck region, sloughing of the lips and oral mucosa, and within the oral cavity covering less than $10 \%$ body surface area. [Figure 1a and 1b]. Nikolsky sign was positive.

The diagnosis of Steven Johnson Syndrome was made on clinical grounds by a dermatological consultation.

His complete blood count suggested pancytopenia (hemoglobin - $10 \mathrm{~g} / \mathrm{dl}$, total leukocyte count - 700/UL, platelet count $-8,000 / \mathrm{UL}$ ). His blood investigation suggested severe neutropenia (Absolute Neutrophil Count $=35$ ). His serum albumin level was noted to be $2.8 \mathrm{~g} / \mathrm{dl}$ and his serum alkaline phosphatase was $306 \mathrm{U} / \mathrm{L}$. His urine routine examination showed $3+$ urine sugar. Other biochemical test results were within normal limits initially.

The patient was admitted to the intensive care unit. Blood culture was sent and the patient was started on supportive antibiotics with injection Vancomycin and Gentamycin. In view of decreased counts, injection filgrastim $50 \mu \mathrm{g}$ was given subcutaneously once a day. The other supportive cares included wound care, fluid and electrolyte management, nutritional support, ocular care, temperature control and pain management.

After stopping the chemotherapy, the patient's rash started to improve but he developed respiratory symptoms which further deteriorated and he succumbed to his illness on the 6th day in spite of all best measures being taken.

\section{DISCUSSION:}

The mechanism underlying SJS still remains unknown in patients administered with combination chemotherapy. In our case, we believed that the patient's problem was related to one of the four chemotherapy drugs since the syndrome was not present before the initiation of chemotherapy, suggesting that it was not part of the presenting features of his disease.

Although SJS occurs in a very small percentage of patients who use chemotherapy drugs, there are several reports reporting a potential association of anticancer drugs with SJS and TEN, including conventional cytotoxic and novel targeted agents. [6] In our case, the last administered drug to the patient was Daunorubicin which according to FAERS (Food and Drug Administration Adverse Effect Reporting System) has very few reported cases of SJS. [6] We believe Daunorubicin to be the culprit for SJS in our patient as the immediate withdrawal of Daunorubicin led to improvement in signs and symptoms.

Daunorubicin forms the cornerstone of treatment in the remission induction of both adult and childhhod acute leukaemia. A liposomal formulation of daunorubicin is available as a first line therapy for advanced HIV related Kaposi sarcoma. It is a cytotoxic antibiotic which acts by intercalating between DNA base pairs and uncoiling the DNA helix, which results in inhibition of DNA synthesis and apoptosis of rapidly dividing cells. Common side-effects of Daunorubicine include myelosuppression, nausea, vomiting, mucositis, diarrhea, alopecia, red urine and cardiotoxicity. Cutaneous side effects are very rare and include reversible alopecia, rash, contact dermatitis and urticaria. [7] The possible association of Daunorubicin with SJS has not been well-established and inconsistently reported.

The main difficulty in attempting to identify an offending agent in chemotherapy that may potentially serve as triggers stems from the frequent co-administration of not only multiple anticancer agents but also drugs that are known to have strong associations with these cutaneous reactions (e.g. sulfonamide antibiotics, corticosteroids, allopurinol). [1-4] In the majority of published case reports patients were exposed to multiple agents.

In our case, the patient was also co-administered corticosteroids as part of the remission induction chemotherapy. Although, there are few reports suggesting corticosteroids as an offending agent of SJS/TEN, their role as triggers is not clearly defined and there is a high potential for confounding by several factors, including the use of anti-infective and anticonvulsant agents, a history of radiotherapy, and a history of collagen vascular disease. $[8,9]$ 
The mucocutaneous complications of anti-cancer drugs are widely observed in oncology and hematology departments, but clinicians/oncologists may overlook these reactions because of the severity of the underlying primary disorder itself. In conclusion, it is very critical but complicated to identify the offending agents when oncologic patients develop SJS during chemotherapyIt is crucial to be able to differentiate life-threatening cutaneous adverse reaction that require immediate management from more benign manifestations of chemotherapy and treat the same as an oncologic emergency. The potential association of Daunorubicin with SJS should be considered and administered with caution. As soon as the first suspicious sign of SJS/TEN is observed, the offending drug must be discontinued immediately, aggressive medical management must be started or the patient must be referred to appropriate centers promptly.

Early recognition and intervention can significantly alter the course of the disease and improve the outcome. Identification and prompt removal of precipitating factors are the most pivotal steps for the lifesaving management of SJS.

\section{CONCLUSION:}

Clinicians should consider the potential association of Daunorubicin with SJS, administer it with caution and observe patients closely for potentially dangerous cutaneous reaction and treat it as an oncologic emergency. If multi-drug regimen chemotherapy is employed, one must administer the drugs with caution and all subsequently developing cutaneous reactions must promptly be evaluated with a high index of suspicion for SJS.

\section{AUTHOR CONTRIBUTION:}

Preeti Shakya: Conceptualization (lead); writing-original draft (lead), Writing-review and editing (equal)

Amit Sharma Nepal : Critical revision of the article (lead), Writing- review and editing (equal)

Both authors contributed to the final version of the manuscript.

\section{REFERENCES:}

1. Castana O, Rempelos G, Anagiotos G, Apostolopoulou C, Dimitrouli A, Alexakis D. Stevens-johnson syndrome: a case report. Ann Burns Fire Disasters. 2009;22(3):147-151.

2. La Grenade L, Lee L, Weaver J, Bonnel R, Karwoski C, Governale L, Brinker A. Comparison of reporting of Stevens-Johnson syndrome and toxic epidermal necrolysis in association with selective COX-2 inhibitors. Drug Saf. 2005;28(10):917-924.

3. Mockenhaupt M, Viboud C, Dunant A, Naldi L, Halevy S, Bouwes Bavinck JN, Sidoroff A, Schneck J, Roujeau JC, Flahault A. Stevens-Johnson syndrome and toxic epidermal necrolysis: assessment of medication risks with emphasis on recently marketed drugs. The EuroSCAR-study. J Invest Dermatol. 2008;128(1):3544.

4.Halevy, S., Ghislain, P. D., Mockenhaupt, M., Fagot, J. P., Bavinck, J. N. B., Sidoroff, A., \& EuroSCAR Study Group. (2008). Allopurinol is the most common cause of Stevens-Johnson syndrome and toxic epidermal necrolysis in Europe and Israel. Journal of the American Academy of Dermatology , 58 (1), 25-32.

5. LiverTox: Clinical and Research Information on Drug-Induced Liver Injury [Internet]. Bethesda (MD): National Institute of Diabetes and Digestive and Kidney Diseases; 2012-. Daunorubicin. [Updated 2017 Dec 27]. Available from: https://www.ncbi.nlm.nih.gov/books/NBK548259/

6. Rosen AC, Balagula Y, Raisch DW, et al. Life-threatening dermatologic adverse events in oncology. Anticancer Drugs . 2014;25(2):225-234

7. Saleem T, Kasi A. Daunorubicin. [Updated 2020 Sep 19]. In: StatPearls [Internet]. Treasure Island (FL): StatPearls Publishing; 2020 Jan-. Available from: https://www.ncbi.nlm.nih.gov/books/NBK559073/ 
8. Mockenhaupt M, Viboud C, Dunant A, Naldi L, Halevy S, Bouwes Bavinck JN, Sidoroff A, Schneck $J$, Roujeau JC, Flahault AJ Invest Dermatol. Stevens-Johnson syndrome and toxic epidermal necrolysis: assessment of medication risks with emphasis on recently marketed drugs. The EuroSCAR-study.2008 Jan; 128(1):35-44.

9. Roujeau, J. C. et al. Medication use and the risk of Stevens-Johnson syndrome or toxic epidermal necrolysis. N. Engl. J. Med. 333, 1600-1607. https://doi.org/10.1056/NEJM199512143332404 (1995).

\section{FIGURE LEGENDS:}

Figure 1: Skin changes in Steven-Johnson Syndrome

(a) Initial erythematous rash with sloughing of mucosa over the angle of mouth

(b) Erythematous morbilliform rash with mucocutaneous involvement in lips, face and neck 


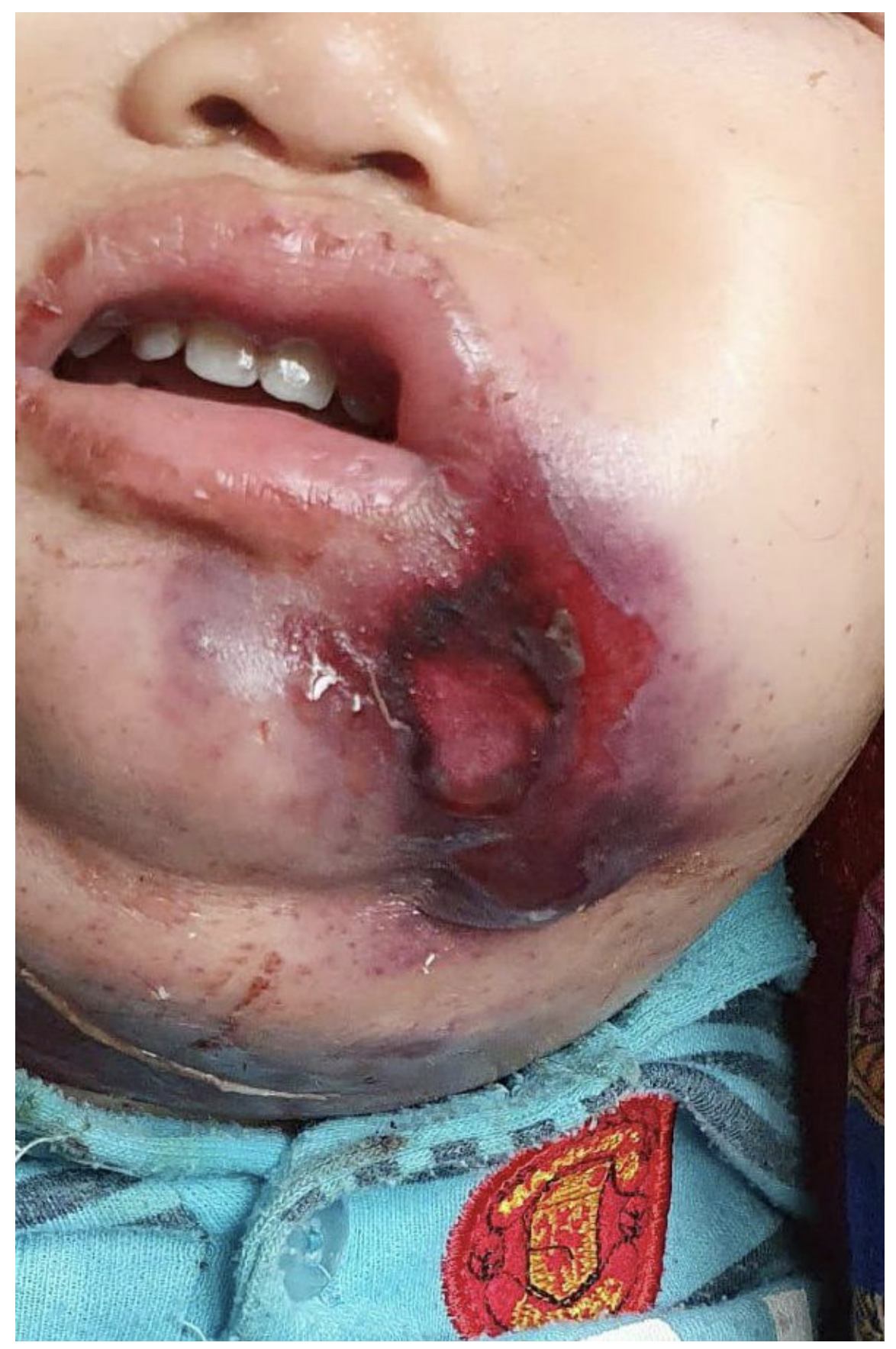




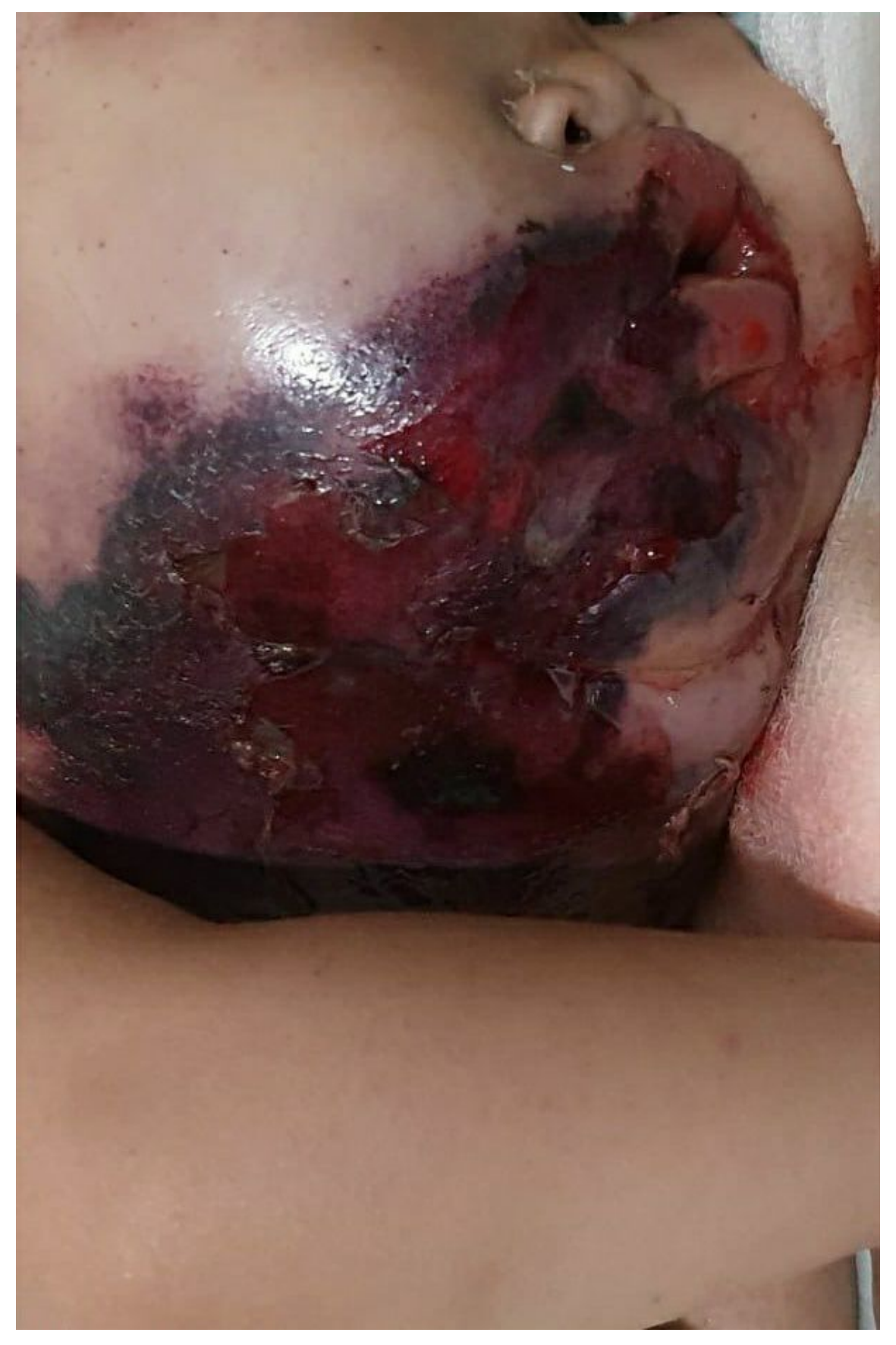

